\title{
Ajudando as crianças a enfrentarem o luto pela perda de pessoas significativas por COVID-19
}

Helping children to mourn the loss of significant people by COVID-19

Ayudar a los niños a llorar la pérdida de personas significativas por COVID-19

Isabella Navarro Silva ${ }^{1}$ (D) https://orcid.org/0000-0001-7551-0150

Amanda Cristine Haddad de Miranda ${ }^{1}$ (D https://orcid.org/0000-0002-8450-4075

Lucas Thiago Pereira da Silva ${ }^{1}$ (D) https://orcid.org/0000-0003-3258-092x

Regina Szylit ${ }^{1}$ (D) https://orcid.org/0000-0002-9250-0250

\section{Resumo}

Trata-se de um artigo teórico de teor reflexivo, no qual propõe-se a reflexão sobre o uso de estratégias lúdicas, como a criação e a contação de histórias, na comunicação com as crianças que perderam pessoas significativas por COVID-19. Diante de diversas mudanças, incertezas e perdas, é indispensável garantir à criança espaços seguros para o diálogo. E para aquele que estabelece o papel de conversar com a criança, torna-se importante ter à sua disposição um instrumento que possibilite a fluidez da comunicação acerca de temas delicados, como a morte e 0 luto, de forma a permitir que a criança expresse suas dúvidas, seus medos e suas angústias em uma abordagem que esteja centrada no cuidado à criança e à família.

\section{Abstract}

This is a theoretical article with a reflective content, in which it is proposed to reflect on the use of playful strategies, such as creation at stories and storytelling, in communicating with children who lost significant people through COVID-19. In the face of various changes, doubts and losses, it is essential to ensure the child safe spaces for dialogue. And for one who establishes the role of talking to the child, it is important to have at their disposal an instrument that allows the fluidity of communication about delicate themes, such as death and grief, in a way that allow that the child expresses her doubts, her fears and her anguish in an approach that centered on child and family care.

\section{Resumen}

Es un artículo teórico con un contenido reflexivo, en el que se propone reflexionar sobre el uso de estrategias lúdicas, como la creación de historias y la narración, en la comunicación con los niños que perdieron personas significativas por COVID-19. Ante diversos cambios, incertidumbres y pérdidas, es esencial garantizar al niño espacios seguros para el diálogo. Y para aquellos que establecen el papel de hablar con el niño, es importante tener a su disposición un instrumento que permita la fluidez de la comunicación sobre temas delicados, como la muerte y el aflicción, de una manera que posibilita el niño exprese sus dudas, miedos y angustias en un acercarse que se centra en el cuidado infantil y familiar.

\section{Descritores}

Enfermagem pediátrica; Criança; Luto;

Coronavirus; Infecções por coronavirus; COVID-19

\section{Keywords}

Pediatric nursing; Child; Bereavement; Coronavirus; Coronavirus infections; COVID-19

\section{Descriptores}

Enfermería pediátrica; Niño; Aflicción; Coronavirus; Infecciones por coronavirus; COVID-19

\section{Como citar}

Silva IN, Miranda AC, Silva LT, Szylit R. Ajudando as crianças a enfrentarem o luto pela perda de pessoas significativas por COVID-19. Rev Soc Bras Enferm Ped. 2020;20(Especial COVID-19):85-90.

${ }^{1}$ Escola de Enfermagem, Universidade de São Paulo, São Paulo, SP, Brasil.

Conflitos de interesse: nada a declarar.

Submetido: 31 de Julho de 2020 | Aceito: 18 de Setembro de 2020

Autor correspondente: Isabella Navarro Silva | E-mail: isabella.navarro.silva@usp.br

DOI: http://dx.doi.org/10.31508/1676-3793202000000129 


\section{Introdução}

A morte e suas temáticas adjacentes, tais como a perda e o luto, são processos humanos que tendem a ser geralmente negados, tanto pelos indivíduos que os vivenciam como também por aqueles que não os vivenciam, mas que têm de lidar com esses processos de alguma maneira, sendo essa negação potencializada quando o contexto é vivenciado por crianças ou quando está relacionado a elas..$^{(1)} \mathrm{O}$ fato de esses assuntos serem pouco explorados os torna um tabu em conversas entre os adultos, o que é transferido e ganha força quando são tratados com as crianças, uma vez que comumente a infância e a finitude da vida são aparentemente duas realidades contraditórias. ${ }^{(2)}$ Entretanto, as discussões sobre doença, morte e luto ganham cada vez mais destaque nos últimos meses.

Desde março de 2020, a Organização Mundial da Saúde (OMS) vem afirmando que a COVID-19 - doença causada pelo Sars-CoV-2 (novo coronavírus) - é caracterizada como uma pandemia, ${ }^{(3)}$ tendo, entre seus desdobramentos, uma crise de saúde pública, o estabelecimento de distanciamento social e as mudanças na forma de viver e se relacionar. São diferenças que impactam também na saúde mental das crianças, que têm experimentado perdas repentinas e abruptas em várias áreas da vida, tendo que adaptar-se a novas rotinas e dinâmicas, com o ambiente de suas casas sendo transformado em locais para trabalho para os pais, e de atividades escolares, de lazer e de descanso. ${ }^{(4)}$

Na vigência de um isolamento social, as crianças não podem frequentar locais públicos, de maior circulação e interação social, ou visitar pessoas com as quais estavam habituadas a se relacionar frequentemente avós, tios, primos e amigos - por longos períodos. Em alguns casos, essas mudanças são acompanhadas da morte de um familiar próximo em decorrência da COVID-19. Nesse contexto, as crianças ainda se deparam com mudanças nos rituais envolvidos no processo de despedida, que, devido ao novo cenário, tiveram de ser ressignificados e ganhar nova forma e novo conteúdo. Ou seja, situações novas que impactam na maneira como o indivíduo enxerga o mundo e lida com o que está vivendo.

Os sentimentos da família no processo de morrer de um de seus membros são os mais diversos possíveis, sendo os familiares invadidos por uma dor profunda quando tomam ciência da situação de fim de vida do ente próximo - processo esse que pode ser vivenciado e partilhado com a criança enquanto membro da unidade familiar. ${ }^{(5)}$ Sendo a perda, a morte e o luto uma situação possível e factível para a criança, que pode ter seu relacionamento, seus sonhos e/ou seu projeto de vida rompidos repentinamente e abrupta, é necessário que se estabeleça uma comunicação aberta e segura no ambiente familiar - que precisa estar preparado para oferecer espaço e tempo para as expressões de tristeza, culpa e raiva da criança, de modo que ela possa contar com ouvintes dispostos para acolhê-la. ${ }^{(6)}$ Para dialogar com a criança a respeito do tema, é necessário que seja levado em consideração que o comunicador tenha um olhar atento e respeitoso ao desenvolvimento cognitivo e emocional dela, além de uma postura flexível, prudente e protetora. ${ }^{(6)}$

Diante de diversas mudanças, incertezas e perdas, é indispensável garantir à criança espaços seguros de comunicação, nos quais ela possa manifestar o que conhece, por meio da linguagem verbal ou da linguagem não verbal. ${ }^{(5)}$ Isso pode ser feito através de histórias e desenhos cujos temas tragam possibilidades para serem vivenciadas pela criança nesse contexto. Para facilitar o processo de compressão e atribuição de conceito e significado, deve-se considerar a faixa etária da criança, bem como o estágio do desenvolvimento infantil em que se encontra, uma vez que ela pode se apropriar das formas indiretas de falar sobre o assunto, utilizando-se, por exemplo, da literatura infantil.(7)

Jogos, brincadeiras e atividades lúdicas são instrumentos relevantes para o processo de aprendizagem, uma vez que, quando bem direcionados, estimulam a concentração, desenvolvimento da linguagem, senso de coletividade e cooperação, além da capacidade de assimilação, possibilitando a construção valores e superação de desafios. Por meio do uso do lúdico a criança tem a oportunidade de repetir situações traumáticas, dolorosas e conflitantes, contribuindo para que ela consiga, de alguma maneira, elaborá-las e ressignificá-las. ${ }^{(8,9)}$

Assim sendo, no presente artigo, propõe-se a reflexão sobre o uso de estratégias lúdicas, como a criação e a contação de histórias, na comunicação com as crianças que perderam pessoas significativas por $\mathrm{CO}$ VID-19, tendo em vista que é importante, para aquele que estabelece o papel de conversar com as crianças, 
ter à sua disposição um instrumento que possibilite a fluidez da comunicação acerca de temas delicados, como a morte, o luto e a perda, de modo que as crianças tenham a oportunidade de expressar suas dúvidas, seus medos e suas angústias em uma abordagem que esteja centrada no cuidado à criança e à família.

\section{Métodos}

Trata-se de um artigo teórico de teor reflexivo, baseado em estudos e discussões realizadas pelo Núcleo Interdisciplinar de Pesquisa em Perdas e Luto, da Escola de Enfermagem da Universidade de São Paulo. Fundado em 2007, esse núcleo dedica-se à produção de conhecimentos relativos ao cuidado do paciente e sua família, à morte e luto e aos cuidados paliativos. Desde 2018, tem se aprofundado também em questões que envolvam conjuntamente os eixos: a) criança e sua família, b) morte e luto e c) comunicação através de estratégias lúdicas.

As reflexões propostas foram embasadas na literatura nacional e internacional referente ao tema, além da experiência dos autores na prática, no ensino e na pesquisa. E, principalmente, são desdobramentos das atividades atreladas aos projetos em andamento - levantamentos bibliográficos e busca ativa da literatura referente aos temas; formação complementar específica para criação e contação de histórias; elaboração e adaptação de estratégias lúdicas e desenvolvimento de oficinas envolvendo criação e contação de histórias com crianças de diferentes faixas etárias, em parques e em instituições de ensino e de saúde.

A apresentação das explanações e reflexões se dará na forma de eixos condutores sobre o tema, advindos de interpretações da literatura e, também, de impressões reflexivas dos autores. Como não houve nenhuma interação de pesquisa com seres humanos, para sua elaboração, não foi necessário submeter este estudo a trâmites éticos.

\section{Morte e luto para a criança}

Embora testemunhar a morte seja parte da vida, sendo a única certeza que temos, deparar-se com ela provoca nas pessoas sentimentos de incapacidade, fragilidade e tristeza. ${ }^{(1,10)}$ É um acontecimento que afeta os indivíduos em todos os âmbitos - afetivo, físico, comportamental, social e espiritual -, estando relacionado com a difícil realidade de separar-se e de aprender a viver em um mundo sem uma determinada pessoa.

Como consequência dessa separação, tem-se o luto - um processo dinâmico e multidimensional, pelo qual o indivíduo que sofreu a perda de algo significativo atravessa. ${ }^{(10)} \mathrm{E}$, ao longo desse luto, será vivenciada uma série de mudanças, relacionadas aos meios social, familiar e econômico, entre outras, associadas diretamente a como o enlutado experiência o processo de perda. ${ }^{(11)}$

Todas essas manifestações - de sentimentos, questionamentos e expressões -, relacionadas à perda e à morte, atingem também as crianças. No entanto, por ser um conceito complexo para ser entendido e por pressupor deparar-se com a própria finitude, o tema tende a ser evitado, sobretudo quando a conversa é com uma criança. ${ }^{(12)} \mathrm{O}$ fenômeno da morte é um enigma indecifrável em sua totalidade, considerado um evento estressante tanto para os adultos quanto para as crianças. Por esse motivo, muitos pais e cuidadores mantêm as trocas de informação sobre esse assunto em um nível mínimo com as crianças, utilizando, recorrentemente, explicações ambíguas, metafóricas e/ou espirituais e acreditando ser esta uma forma de proteger e oferecer segurança para elas. ${ }^{(5,13)}$

Entretanto, o silêncio, a negação ou a utilização de explicações abstratas acerca da morte podem prejudicar o crescimento global da criança, além de confundir o pensamento dela, ao invés de lhe proporcionar conforto e clareza para a circunstância da perda. Ademais, negligenciar o assunto, pode gerar sofrimento emocional, como ansiedade, confusão, medo, estresse e percepções errôneas a respeito da temática morte, perda e luto. ${ }^{(14)}$

É comumente observada a discussão do quanto o conceito de morte é diretamente influenciado pelos aspectos sociais, culturais e espirituais. Todavia, tem-se o consenso de que esse conceito é construído a partir dos significados de subconceitos - sendo a universalidade, a irreversibilidade, a inevitabilidade e a não funcionalidade os principais. ${ }^{(5,6,15)}$ Para encontrar a melhor maneira de esclarecer em que consiste a morte, é fundamental observar em que momento do desenvolvimento cognitivo e emocional a criança está 
- entendendo que a abordagem não pode ser a mesma a depender do estágio de desenvolvimento que ela se encontre. ${ }^{(16)}$

Seguindo os estágios estabelecidos por Jean Piaget, é possível encontrar discussões sobre a aquisição do conceito de morte pelas crianças. ${ }^{(5,6)}$ No período chamado sensório-motor, o conceito de morte não existe: as crianças percebem a morte como ausência e falta. No período pré-operacional, as crianças relacionam a morte à imobilidade, mas tendem a pensar na morte como um fenômeno temporário e reversível, não entendendo-a como uma ausência sem retorno. Já, no período operacional concreto, elas entendem a oposição entre a vida e a morte, compreendendo a morte como um processo definitivo e permanente; as crianças ainda não são capazes de explicar adequadamente as causas da morte, mas conseguem apreender o conceito de morte em sua totalidade - em relação à não funcionalidade, à irreversibilidade e à inevitabilidade da morte. Por fim, no período operacional formal, o conceito de morte torna-se mais abstrato, as crianças compreendem a morte como inevitável, universal, irreversível e pessoal, utilizando explicações de ordem natural, fisiológica e teológica.

Falar de morte para crianças tende a representar uma grande dificuldade para os adultos, que muitas vezes são questionados sobre o que é a morte, se há vida para além dela e o que acontece às pessoas que morreram. No entanto, o estabelecimento do diálogo em um contexto de morte e luto se faz fundamental. Ao facilitar a expressão das manifestações e dos sentimentos da criança, o adulto dá a ela a possibilidade de manejar seus medos e fantasias sobre a morte e, consequentemente, passa a representar um apoio, para a criança, no processo de elaboração do seu luto. ${ }^{(15,17)}$

Estudiosos e especialistas em luto enfatizam a importância de se conversar com a criança sobre a morte de maneira honesta e informativa, retratando-a como parte do ciclo de vida. ${ }^{(12)}$ Sendo algo natural, a morte pode estar presente nas histórias e brincadeiras, no trabalho da escola, nas discussões durante o jantar, nos questionamentos que fazemos para aqueles em quem acreditamos e confiamos. Assim, para além de discutir sobre a morte e suas questões, trata-se da criação de um vínculo, um espaço que permita à criança expor seus sentimentos e angústias perante situações desconfortáveis e desconhecidas.

\section{Os recursos lúdicos como estratégia para ajudar as crianças que sofreram perdas significativas e a atuação da enfermagem}

O brincar é de fundamental importância para o desenvolvimento infantil, uma vez que estimula a criança a se organizar no tempo, a treinar suas habilidades motoras e a conhecer suas vontades e pensamentos, além de promover a construção de relacionamentos seguros e estimulantes. ${ }^{(18)}$ Ao utilizar este recurso, a criança é capaz de criar uma bolha lúdica em sua volta, onde se sente segura, acolhida pelo mágico e capaz de realizar suas fantasias. Mas, para que isso ocorra, é fundamental que ela esteja em um ambiente adequado para sua faixa etária e com pessoas que reconheçam a importância da brincadeira e valorizem este processo.

O termo "ludicidade" tem origem no latim "ludus" e significa jogo, exercício ou imitação, ${ }^{(19)}$ o que realizamos por meio de brinquedos e brincadeiras, que envolvam teatro, literatura, música, entre outros. Além de incentivar a imaginação e proporcionar momentos de diversão, o lúdico possui capacidade de educar, desenvolver a percepção do mundo, prover interações sociais e enriquecer o conhecimento de quem brinca.

A criação e a contação de histórias são instrumentos lúdicos que auxiliam na comunicação com a criança, podendo contribuir para o enfrentamento de suas perdas, suas angústias e seus medos. Por meio desses instrumentos, podemos abordar as temáticas perda, morte e luto com crianças de diferentes idades, tanto em hospitais quanto em escolas.

Utilizando a literatura infantil disponível, a partir da leitura de histórias, a criança pode se identificar com alguns personagens, por suas experiências emocionais e situações vividas serem parecidas com as narrativas literárias. ${ }^{(13)}$ Assim, a contação de história pode contribuir para o enfrentamento de eventos difíceis em um espaço onde a criança se sinta segura, podendo ser um recurso utilizado por todas as pessoas que, de alguma forma, prestam cuidados à criança. ${ }^{(13)}$ Nesses cenários lúdicos, a criança transparece sua história e nos permite acessar o material de seu inconsciente. ${ }^{(9)}$ 
Em atividades de pesquisa e extensão desenvolvidas, observa-se a assiduidade das crianças em colaborar e participar de oficinas que envolvam o criar e o contar histórias. Elas são capazes de associar o tema das histórias contadas com eventos experienciados em seu dia a dia, fato geralmente evidenciado por comentários feitos durante ou após as atividades e que abordavam situações semelhantes ou projeções de sentimentos. Entretanto, em alguns momentos, é possível que a criança solicite outra história ou interrompa o jogo - e isto não significa que a criança não esteja trabalhando inconscientemente o assunto, uma vez que é pela imaginação e fantasia que a criança reflete sobre o mundo real e busca maneiras de confrontá-lo. ${ }^{(13)}$

Através do uso de instrumentos lúdicos, as crianças fazem comparações entre as histórias contadas e a perda de animais de estimação, parentes e até colegas de escola, além de se sentirem seguras durante a brincadeira para falar sobre o câncer, a depressão, a COVID-19 ou até mesmo sobre como são realizados enterros. Nesse sentido, as situações descritas corroboram a ideia de que o brinquedo passa a representar simbolicamente um sistema de fala, no qual a criança pode se ancorar para exteriorizar e ressignificar a realidade vivenciada. ${ }^{(2,18)}$ Por isso, nesses espaços de discussão, é fundamental acolher as dúvidas e os sentimentos da criança, além de traduzir possíveis metáforas empregadas.

As estratégias lúdicas podem ser utilizadas como ferramenta importante para abrir um espaço seguro de diálogo sobre o novo coronavírus, a pandemia e suas consequências biopsicossociais. Estudos mostram que o senso de segurança e normalidade das crianças está passando por mudanças drásticas - elas testemunham a perda de emprego confiável de seus familiares, ouvem sobre a saúde vulnerável de seus avós e percebem que o novo vírus está ultrapassando estruturas e sistemas que antes pareciam seguros na sua visão de mundo - o que implica na necessidade ainda maior da comunicação aberta e acolhedora. ${ }^{(4,20)}$

Criar um ambiente, incluindo um espaço e local confortável, para a criança se sentir segura significa ouvir e responder ativamente às suas perguntas com respostas honestas e apropriadas para a idade e o desenvolvimento dela. Estar aberto e compartilhar informações pode reduzir a ansiedade, a confusão e as percepções errôneas. ${ }^{(20)}$
Nesse contexto, a atividade de cunho lúdico pode estabelecer-se como um recurso facilitador para as intervenções dos profissionais, uma vez que, no processo de trabalho da equipe de enfermagem, além do atendimento das necessidades biológica e fisiológica da criança, é preciso que se dê também atenção aos aspectos psicossociais, dela e de sua família, visando proporcionar o cuidado integral e holístico. ${ }^{(21)}$

A equipe de enfermagem precisa reconhecer as particularidades e os benefícios proporcionados pelo brincar e pela inclusão da atividade lúdica na vivência da criança, prevendo a sua realização, com ela e sua família, de forma a proporcionar meios para a sua execução e sua incorporação na sistematização da assistência de enfermagem oferecida, compreendendo o lúdico como ferramenta eficaz e disponível para o cuidado. Assim, o lúdico pode ser visto como uma ferramenta terapêutica apta para dar prosseguimento e promover o desenvolvimento infantil, como também para auxiliar a criança a melhor compreender o momento peculiar que está vivendo. ${ }^{(22)}$

Ademais, destaca-se que o cuidar brincando ao ser utilizado no dia a dia da enfermagem - propicia uma abordagem integral no cuidado prestado à criança e à família, enfatizando a humanização da assistência. ${ }^{(23)} \mathrm{O}$ lúdico deve ser compreendido como instrumento facilitador, a ser utilizado nas diferentes frentes de atuação da profissão, visando explorar os benefícios e as potencialidades que o cuidar brincando proporciona à criança e ao profissional. ${ }^{(23)}$

\section{Considerações finais}

A experiência da doença grave e da morte interfere na estrutura familiar e, também, nos relacionamentos que, de alguma forma, estão vinculados a esse cenário. Cada um experimenta esse processo de uma maneira diferente. Tendo em mãos um instrumento que possibilite a comunicação entre adultos e crianças sobre o tema delicado que é a morte, o luto e as perdas significativas por COVID-19, pode-se, ao mesmo tempo, abrir espaço para que as crianças expressem suas dúvidas, suas angústias e seus medos e permitir que os adultos dialoguem com os menores de modo mais adequado à nova conjuntura que estão vivendo. Atividades lúdicas viabilizam condições de diálogos 
com crianças, tornando-se estratégias potenciais para a comunicação.

Assim, como meios lúdico, singular e interativo, a criação e a contação de histórias configuram-se como importantes aliados dos adultos na abordagem de temas diversos com crianças, sobretudo perda e luto. Entendemos que a criança deva sentir-se confortável para falar sobre morte e que isso deve ocorrer no tempo dela. Insistir no assunto, uma vez que a criança não esteja aberta para discuti-lo, pode acarretar mais traumas, provocando-lhe maior dificuldade de se expressar e enfrentar seus medos. Sendo assim, a equipe de enfermagem desempenha papel indispensável no cuidado do paciente e sua família e na comunicação entre eles. É por meio da interação e do vínculo, que se formam entre as partes, que o enfermeiro torna-se capaz de entender as necessidades do grupo e de buscar meios para trabalhar essas necessidades, tendo, na possibilidade da utilização de atividades lúdicas, uma potencial ferramenta no processo de trabalho da equipe no cuidado prestado à criança e a seus familiares no contexto de morte e luto por causa da pandemia de COVID-19.

\section{Referências}

1. Silva IN, Salim NR, Szylit R, Sampaio PS, Ichikawa CR, Santos MR. Knowing nursing team care practices in relation to newborns in end-of-life situations. Esc Anna Nery. 2017;21(4)::20160369.

2. Barbato $K B$, Antunes $K R$, Lourenço MT. Reflexões sobre vivências da criança com câncer diante da morte. Rev SBPH (Belo Horizonte). 2019;22(1):306-27.

3. Organização PanamericanadeSaúde(OPAS).0MSafirmaqueCOVID-19éagoracaracterizada como pandemia. Brasília (DF):OPAS; 2020 [citado 2020 Set 23]. Disponível em: https:// www.paho.org/bra/index.php?option=com_content\&view=article\&id=6120:oms-afirmaque-covid-19-e-agora-caracterizada-como-pandemia\&ltemid $=812$

4. Golberstein E, Wen H, Miller BF. Coronavirus disease 2019 (COVID-19) and mental health for children and adolescents. JAMA Pediatr. 2020;174(9):819-20.
5. Panagiotaki G, Hopkins M, Nobes G, Ward E, Griffiths D. Children's and adults' understanding of death: Cognitive, parental, and experiential influences. J Exp Child Psychol. 2018;166:95115.

6. Souza AS, Oliveira JH. A criança diante da morte: desafios. Rev Estud Interdisc Psicol. 2018;9(1):57-10.

7. Jalmsell L, Kontio T, Stein M, Henter Jl, Kreicbergs U. On the child's own initiative: parents Communicate with their dying child about death. Death Stud. 2015;39(1-5):111-7.

8. Silva RM, Cardozo OG. 0 lúdico como ferramenta facilitador no processo ensino aprendizagem. Rev Cient Inic Invest. 2018; 3(1):85-96.

9. Yogman M, Garner A, Hutchinson J, Hirsh-Pasek K, Golinkoff RM. The power of play: a pediatric role in enhancing development in youn. Pediatrics. 2018;142(3):e20182058.

10. Breen $\mathrm{U}$, Szylit R, Gilbert RK, Macpherson C, Murphy I, Nadeau JW, et al. Invitation to grief in the family context. Death Stud. 2018;1:1-10.

11. Braz MS, Franco MH. Profissionais paliativistas e suas contribuições na prevenção de luto complicado. Psicologia (Cons Fed Psicol). 2017;37(1):90-105.

12. Longbottom S, Slaughter V. Sources of children's knowledge about death and dying. Philos Trans R Soc Lond B Biol Sci. 2018;373:20170267.

13. Arruda-Colli MN, Meaghann SW, Lori W. Communication about dying, death, and bereavement: A systematic review of children's literature. J Palliat Med. 2017;20(5):548-59.

14. Pham S, Porta G, Biernesser C, Payne MW, lyengar S, Melhem N, et al. The burden of bereavement: early-onset depression and impairment in youths bereaved by sudden parental death in a 7-year prospective study. Am J Psychiatr. 2018. https://doi. org/10.1176/appi.ajp.2018.17070792.

15. Oliveira LI, Rocha MA. Conversando sobre morte com a criança em fase terminal. Itabuna, BA; 2016 [citado 2020 Jul 27]. Disponível em: https://www.psicologia.pt/artigos/textos/ A1097.pdf

16. Yang S, Park S.A Sociocultural approach to children's perceptions of death and loss. Omega (Westport). 2017;76(1):53-77.

17. Hirooka K, Otani H, Morita T, Miura T, Fukahori H, Aoyama M, et al. End-of-life experiences of family caregivers of deceased patients with cancer: A nation-wide survey. Psychooncology. 2017;27(1):272-8.

18. Weisberg DS, Hirsh-Pasek K, Golinkoff RM, Kittredge AK, Klahr D. Guided play: principles and practices. Curr Dir Psychol Sci. 2016;25(3):177-82.

19. Massa MS. Ludicidade: da etimologia da palavra à complexidade do conceito. Aprender Cad Filosofia Psicol Educ. 2015; 9(15):111-30.

20. Meaghann S, Weaver MD, Lori W. Applying palliative care principles to communicate with children about COVID-19. J Pain Symptom Manage. 2020;60(1):e8-e11.

21. Mooney-Doyle K, Santos MR, Szylit R, Deatrick JA. Parental expectations of support from healthcare providers during pediatric life-threatening illness: A secondary, qualitative analysis. J Pediatr Nurs. 2017;36:163-72.

22. Regehr $\sqcup$, Heath MA, Jackson AP, Nelson D, Cutrer-Párraga EA. Storybooks to facilitate children's communication following parental suicide: paraprofessional counselors' perceptions. Death Stud. 2019:1-10.

23. Marques EP, Garcia TM, Anders JC, Luz JH, Rocha PK, Souza S. Lúdico no cuidado à criança e ao adolescente com câncer: perspectivas da equipe de de enfermagem. Esc Anna Nery. 2016;20(3):e20160073. 